\title{
Performance of Fibre-Reinforced Slag-Based Alkali Activated Mortar in Acidic Environment
}

\author{
Priyadharshini Perumal ${ }^{1}$, Tirthankar Paul ${ }^{2}$, Tero Luukkonen ${ }^{1}$, Juha Röning ${ }^{2}$, Paivo \\ Kinnunen $^{1}$ and Mirja Illikainen ${ }^{1}$ \\ ${ }^{1}$ Inorganic Binders Group, Fibre and Particle Engineering Research Unit (FPERU), University of \\ Oulu, Pentti Kaiteran katu 1, 90014 Oulu, Finland, Priyadharshini.perumal@oulu.fi \\ ${ }^{2}$ Biomimetics and Intelligent System Group, Information Technology and Electrical Engineering, \\ University of Oulu, Pentti Kaiteran katu 1, 90014 Oulu, Finland, tirthankar.paul@oulu.fi
}

\begin{abstract}
The main aim of the work is to study the effect of different fibres (steel, glass and basalt) on resistance of blast furnace slag-based alkali-activated mortar in acidic environment. The alkaliactivated slag mortars were exposed to 5\% sulfuric and acetic acid solutions for 30 days. Mass change, compressive strength and microstructural changes were evaluated. In plain mortar, it was observed that $70 \%$ of the strength was retained in acetic acid environment whereas only $20 \%$ of residual strength remains in sulphuric acid environment. FTIR spectroscopy shows the degradation of the matrix, which implies the alkali-activated mortar was more vulnerable in sulphuric acid environment due to its aggressive nature compared to acetic acid. Decalcification and formation of calcium acetate also hinders the further progress of damage in acetic acid attack. Fibres helped in improving the performance of the mortar by holding the matrix together when the degradation occurred in acidic environment. Compared to plain mortar, incorporation of steel fibres exhibited a maximum strength retention of $19 \%$ in acetic acid and $7 \%$ in sulphuric acid, followed by glass and basalt fibres. SEM images clearly show the debonding of fibres and disintegration of matrix in acidic environment, which resulted in strength loss.
\end{abstract}

Keywords: Slag, Alkali Activation, Fibre-Reinforced Mortar, Acid Attack, Durability.

\section{Introduction}

Interaction of cementitious materials with acidic environment is an important factor to be considered for the durability of structures exposed to harsh environment such as industrial waste water, sea water and acid rain. Alkali activated materials (AAM) are alternative cementitious system that is extensively studied in recent years (Mehta and Siddique, 2016). AAM does not involve Ordinary Portland cement (OPC) and can use 100\% industrial side streams as the binding material, making them an energy efficient alternative for construction (Heath et al., 2014). The properties of AAM are mainly affected by the type of binder material used. AAM shows similar mechanical characteristics as that of OPC concrete, however still further studies are needed about its long-term durability performances (Provis et al., 2007). In specific, the chemical attack in aggressive environments like acidic, chloride, sulphate and carbonate, are to be focused.

In OPC concrete, calcium is considered to be the weakest link in the hydration product as it leaches out in acidic environment (Bernal et al., 2012). Though there are published studies on the low solubility and high resistance of AAM in aggressive environments (Aiken et al., 2018; Fernández-Jiménez and Palomo, 2009), there are also limitations in understanding their 
performance in the presence of different type of fibers. In this study, an effort is made to study the effect of sulphuric $\left(\mathrm{H}_{2} \mathrm{SO}_{4}\right)$ and acetic $\left(\mathrm{CH}_{3} \mathrm{COOH}\right)$ acid on alkali activated slag (AAS) mortar with steel, glass and basalt fibers. Mainly, strength retention, microstructural changes associated with the structural damages are focused.

\section{Experimental}

\subsection{Materials}

Alkali activated slag mortar (G1) was made with ground granulated blast furnace slag (GGBFS) which was obtained from Finnsementti, Finland. Granules of anhydrous sodium metasilicate (SS, $1 \mathrm{~mm}$ size) with $\mathrm{SiO}_{2} / \mathrm{Na}_{2} \mathrm{O}$ ratio of 0.9 was procured from Alfa Aesar. SS was mixed with GGBFS in the ratio of 1:9 and co-grinded in a ball mill for 30 mins. Four different fibers were used in this study (Fig. 1). Table 1 lists the properties of these fibers. Fiber reinforced mortar can be identified by a nomenclature that G1S6 represents G1 mix with steel fiber of $6 \mathrm{~mm}$ length. Similarly, G and B were used for glass and basalt fibers, respectively. Additionally, a mix with combination of $6 \mathrm{~mm}$ and $12 \mathrm{~mm}$ steel fibers in the ratio of 1:1 (G1SM) was made. Deionized water was used for mortar production.
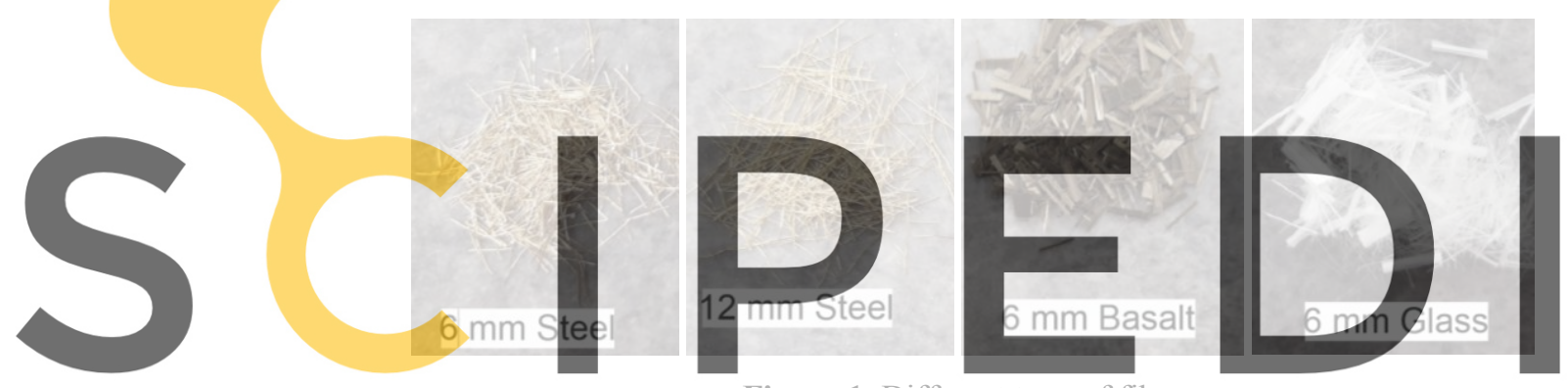

Figure 1. Different type of fibers.

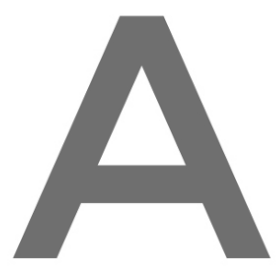

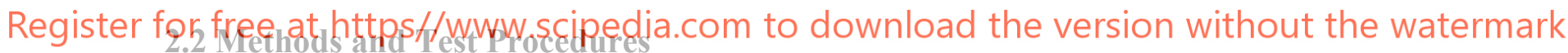

To produce mortar specimens, co-grinded slag and fine aggregate in the ratio of 1:2 was dry mixed for about a minute. Water was added to it (water-binder ratio $=0.35$ ) and further mixed for $3 \mathrm{~min}$. Fibers (1\% weight of binder) were introduced to this slurry gradually while mixing was continued until a homogenous distribution was achieved. The fresh fiber-reinforced alkaliactivated slag mortar was cast into cubic $\left(50 \times 50 \times 50 \mathrm{~mm}^{3}\right)$ molds. The specimens were demoulded after $24 \mathrm{~h}$ and cured in $20{ }^{\circ} \mathrm{C}$ with $100 \% \mathrm{RH}$ for 28 days.

Table 1. Properties of fibers.

\begin{tabular}{|l|r|r|r|r|}
\hline \multirow{2}{*}{\multicolumn{1}{|c|}{ Properties }} & \multicolumn{4}{|c|}{ Type of fibre } \\
\cline { 2 - 5 } & \multicolumn{2}{|c|}{ Steel } & Glass & Basalt \\
\hline Length $(\mathrm{mm})$ & 6 & 12 & 6 & 6 \\
\hline Density $\left(\mathrm{g} / \mathrm{cm}^{3}\right)$ & 7.88 & 7.85 & 2.68 & 2.67 \\
\hline Modulus of Elasticity $(\mathrm{GPa})$ & 200 & 200 & 72 & 85 \\
\hline Tensile strength $(\mathrm{MPa})$ & 2200 & 2000 & 1400 & 3500 \\
\hline
\end{tabular}


Compressive strength before exposure (three cubic specimens) to acidic environment was determined using $3000 \mathrm{kN}$ capacity with a displacement rate of $1.8 \mathrm{~mm} / \mathrm{min}$. Resistance to acid attack was studied by exposing three cubic specimens each in $5 \%$ acetic $(\mathrm{pH} 2.4)$ and sulphuric ( $\mathrm{pH} 0.8$ ) acids for 30 days. Later, compressive strength of the acid attacked specimens were measured. Broken pieces after testing the strength were milled to powder and used for further analysis. Deterioration mechanism of the acids in different mixes were further investigated using X-ray diffraction (XRD), Fourier transform infrared spectroscopy (FTIR) and scanning electron microscopy (SEM).

One of the cubic specimens was reserved for image analysis. Images of the cross-section of the specimen was captured as $1 \mathrm{~cm}$ cubes with optical microscope. Hence 25 images for each mix was analyzed and used to quantify the area of deterioration in the acid medium. In the color-based image analysis, the k-means clustering is an oft-used algorithm (Qi et al., 2016). In one of each partition, the algorithm detects ' $k$ ' centroid from the ' $n$ ' number of datasets (here, pixel value), $\mathrm{k}<\mathrm{n}$. This algorithm is an iterative process which updates its centroid based on squared Euclidean distance of the data from the centroid within the cluster. Concrete is a composite with many particles of different colors and acid attacked region is not uniform in color too. However, the undisturbed core can be identified as a first step. The ' $\mathrm{k}$ ' value has been chosen randomly and subsequently the clusters are arranged in order to minimize the intracluster variances and maximize the inter-cluster variances. The square error function $\left(\boldsymbol{E}_{\boldsymbol{s q}}\right)$ of the algorithm is given by equation (1),

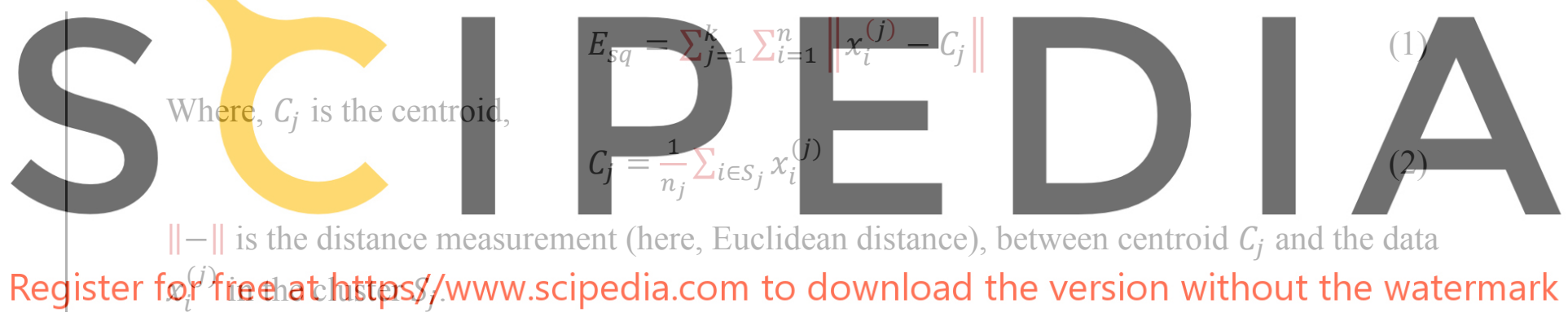

\section{Results and Discussion}

\subsection{Influence of Type of Acid}

Visual examination of the AAS mortar shows very small change in appearance with acetic acid environment, however there is visible disintegration of the specimen when exposed to sulphuric acid (Fig. 2). This is because acetic acid is a weak acid (only partially deprotonated), whereas sulfuric acid is a strong acid (complete deprotonation). Further exploring the chemical/ mineralogical changes on the acid exposed specimens explains that the reaction products formed by interaction of different acids plays an important role. Most important changes of acid attack would be the decalcification of the calcium silicate hydrate $(\mathrm{C}-\mathrm{S}-\mathrm{H})$ in the hydration product. This resulted in shifts in aluminosilicate spectral bands that happens in the region 900 to $1200 \mathrm{~cm}^{-1}$ of FTIR curves of the acid attacked samples (Bakharev, 2005). Further, the formation of calcium acetate with acetic acid is confirmed with the presence of a C-O stretching vibrations at $1556 \mathrm{~cm}^{-1}$ (Fig. 3 (a)). This can also be clearly identified with XRD peaks in Figure 3 (b). The dealumination and condensation caused increase in siloxane chain length and 
corresponding increase in intensity at 980 to $1100 \mathrm{~cm}^{-1}$. This is not significant in sulphuric acid samples, rather shifting of peak at this region was observed. This shows that sulphuric acid resulted in delamination due to depolymerization of the AAS binders.

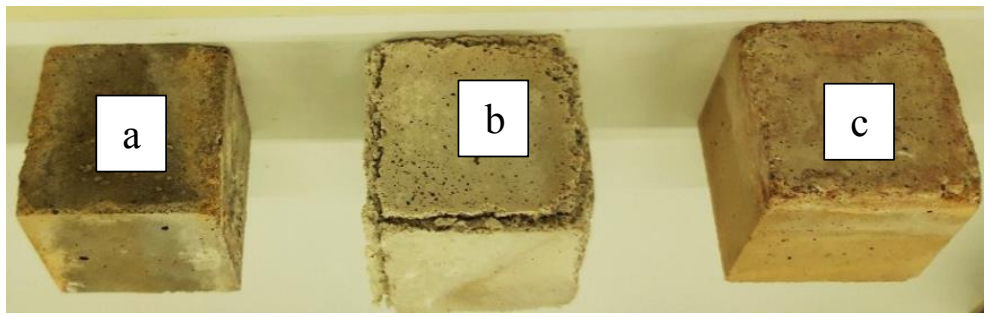

Figure 2. Cubic specimen before (a) and after 30 days exposure in sulphuric (b) and acetic (c) acid.

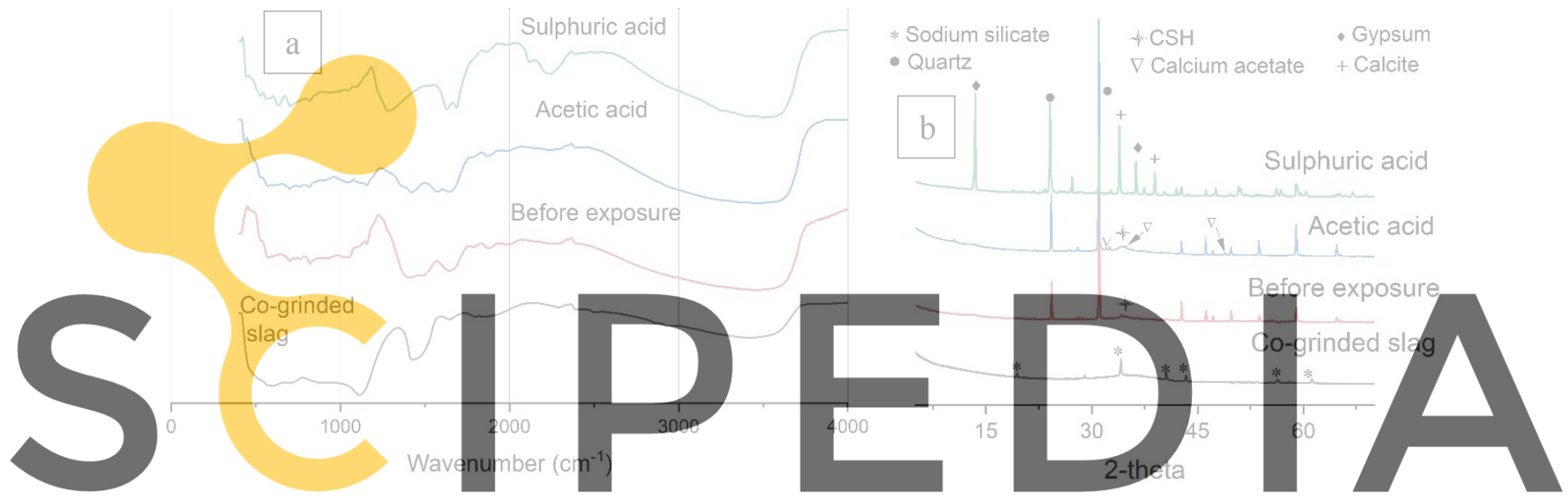

Figure 3. FTIR (a) and XRD (b) spectra of AAS mortar before and after exposure to acid.

Register for free at https//www.scipedia.com to download the version without the watermark SEM images of the acid attacked samples are shown in Figure 3. In both cases, damaged layer and the core area can be identified in the microstructure level. However, it is clearly visible that the sample is vulnerable for debonding in sulphuric acid (Fig. 4 (c)) than in acetic environment (Fig. 4 (a)). The formation of calcium acetate as an intermediate layer (Fig. 5, L2) is well observed in the optical microscopic image of acetic samples. This layer, infact acted as a protection to the core matrix from further attack (Fig. 4 (b)). Though there is also formation of similar layer with sulphuric acid, the reaction products are mainly gypsum and calcite (Fig. 3 (b) and 4 (d)) which were not stable enough to stop the progress of the sulphuric acid in to the core (Koenig and Dehn, 2016; Khan et al., 2018). 

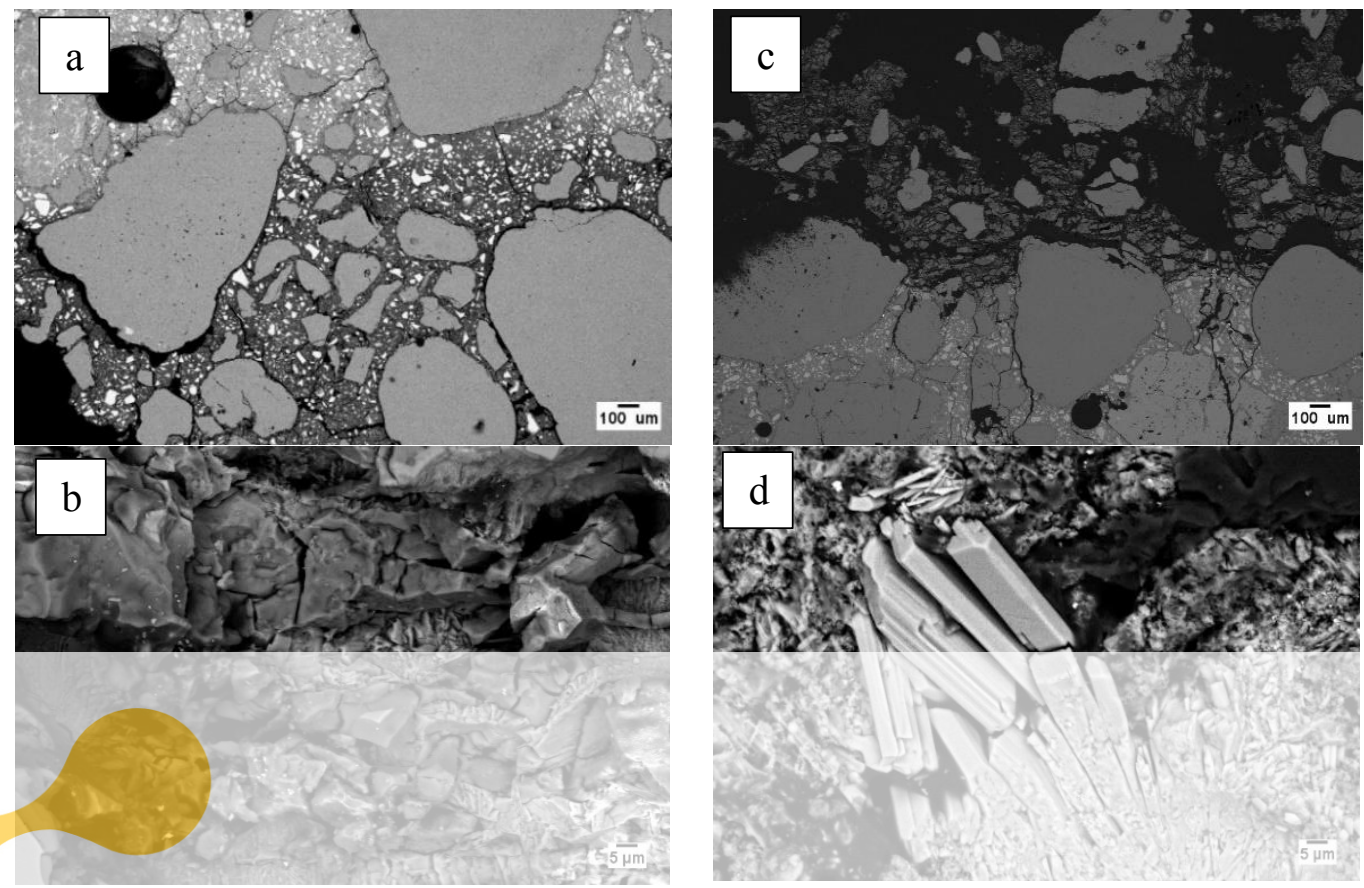

Figure 4. Representative SEM images of AAS mortar exposed in acetic $(\mathrm{a}, \mathrm{b})$ and sulphuric $(\mathrm{c}, \mathrm{d})$

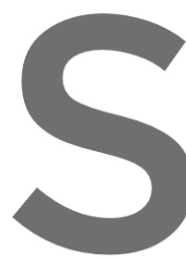

An attempt was made to further quantify the different layers formed and their effec
id medium. However, the damage caused by these acids looked different. Layer
e acetic sample looks denser which could be due to the filling up of pores with acetate. Layer L1 in both the samples looks dis content.

Register for free at https//www.scipedia.com to download the version without the watermark

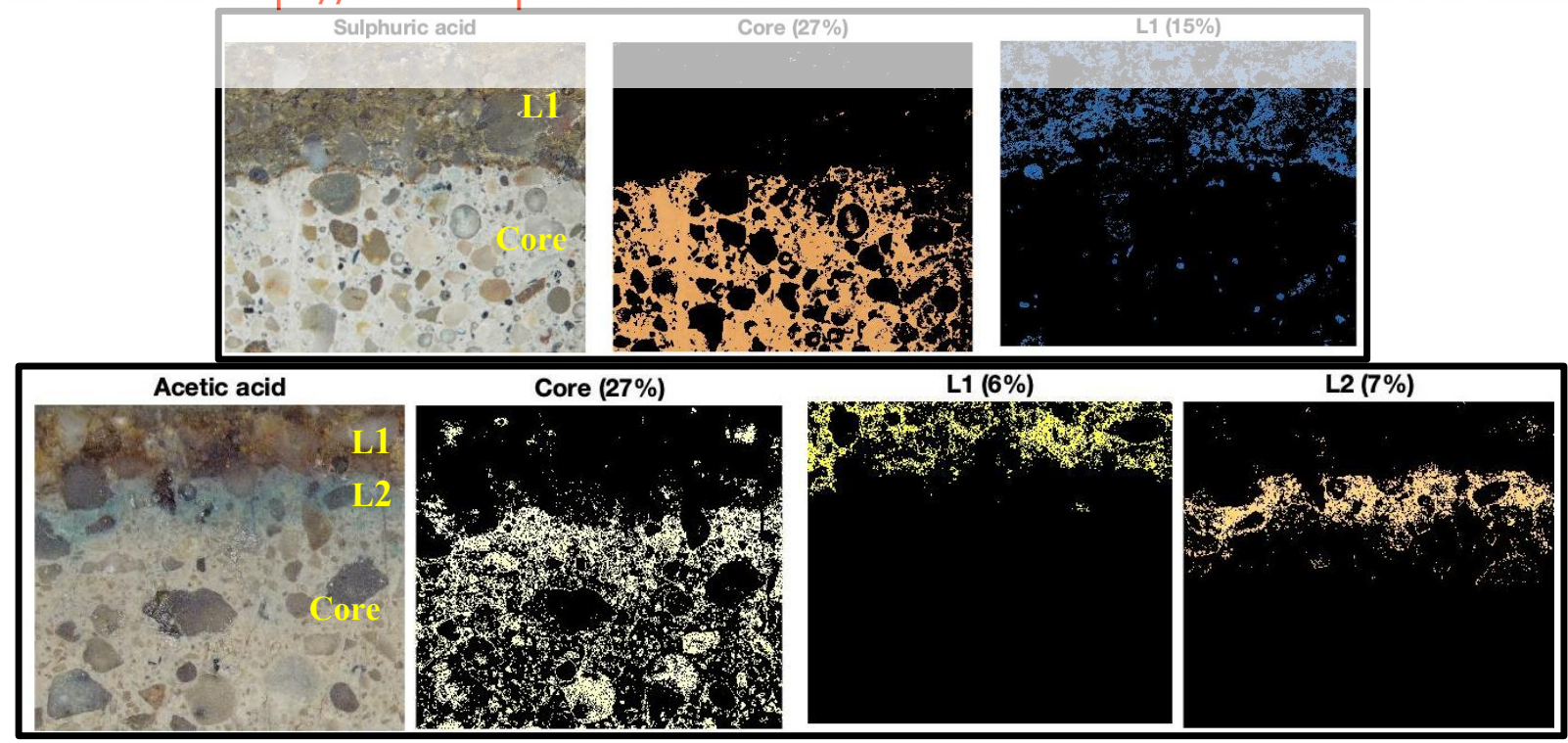

Figure 5. Representative image analysis of acid attacked AAS mortar specimens. 


\subsection{Role of Fiber and Their Type}

Figure 6 (a) displays the effect of fibers on the compressive strength of AAS mortar before and after exposed to acetic and sulphuric acid. It also indicates the percentage reduction in strength with different mix in acidic medium. The addition of steel fibers increased the compressive strength of AAS mortar significantly whereas it was not the case with glass and basalt fibers. Strength reduction with acetic acid exposure is within $10 \%$ for the fiber reinforced AAS mortar except basalt fiber. This could be due to the use of uncoated basalt fiber which might have been damaged in the high alkaline medium (Wei et al., 2010). However, SEM microstructure shows the presence of basalt fibers in the matrix of the acetic attacked samples (Fig. 9 (b). Residual strength of the specimens corroded by acetic acid depends on the fiber used and it is in the order, mixed steel $>6 \mathrm{~mm}$ steel $>6 \mathrm{~mm}$ glass $>12 \mathrm{~mm}$ steel $>6 \mathrm{~mm}$ basalt. This could also be related to the unaffected area and area of the second layer (L2) which protects the core unaffected area from further corrosion (Fig. 6 (b)). SEM images of the acetic affected mixes (Fig. 9 (b), (e), (h)) shows similar structural damage as that of plain AAS mortar (Fig. 4 (a)). The improvement in residual strength of fiber reinforced AAS mortar could be due to the physical action of fibers in holding the disintegrated binder phase to some extent (Kim et al.,

\section{5).}

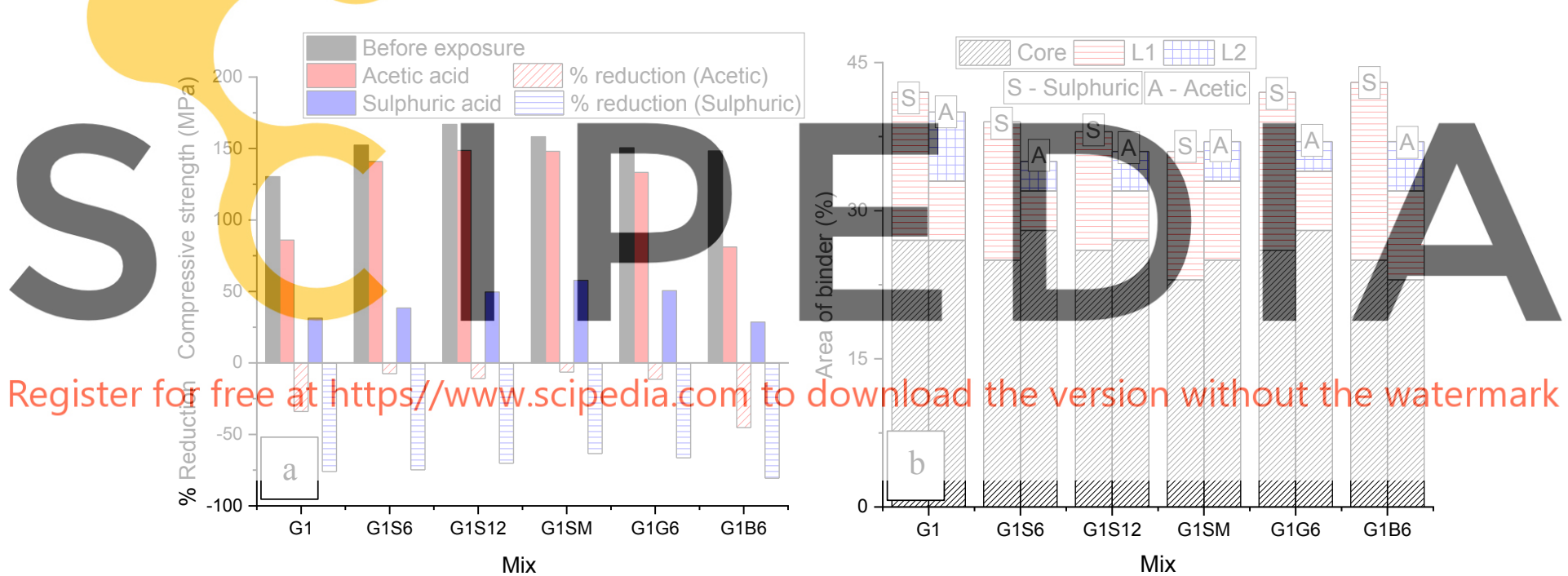

Figure 6. Compressive strength (a) and acid affected area (b) of alkali activated slag mortar.

As mentioned earlier, the effect of sulphuric acid on the AAS mortar was detrimental which is also the case with fiber reinforced AAS mortar. However, with incorporation of fibers, the residual strength improved in the corroded specimens irrespective of the type of acid. The order of resistance to sulphuric acid attack by fibers are, mixed steel steel $>6 \mathrm{~mm}$ glass $>12 \mathrm{~mm}$ steel $>6 \mathrm{~mm}$ steel $>6 \mathrm{~mm}$ basalt. This order is also maintained in the unaffected area (core) as identified in image analysis (Fig. 6 (b)). It can be seen from the SEM images that sulphuric acid corroded the sample and debonded the fibers completely (Fig. 9 (c), (f), (i)). The basalt fibers could not be even located in the corroded samples, making it clear that they need to be treated for such harsh environments (Fig. 9 (i)). 

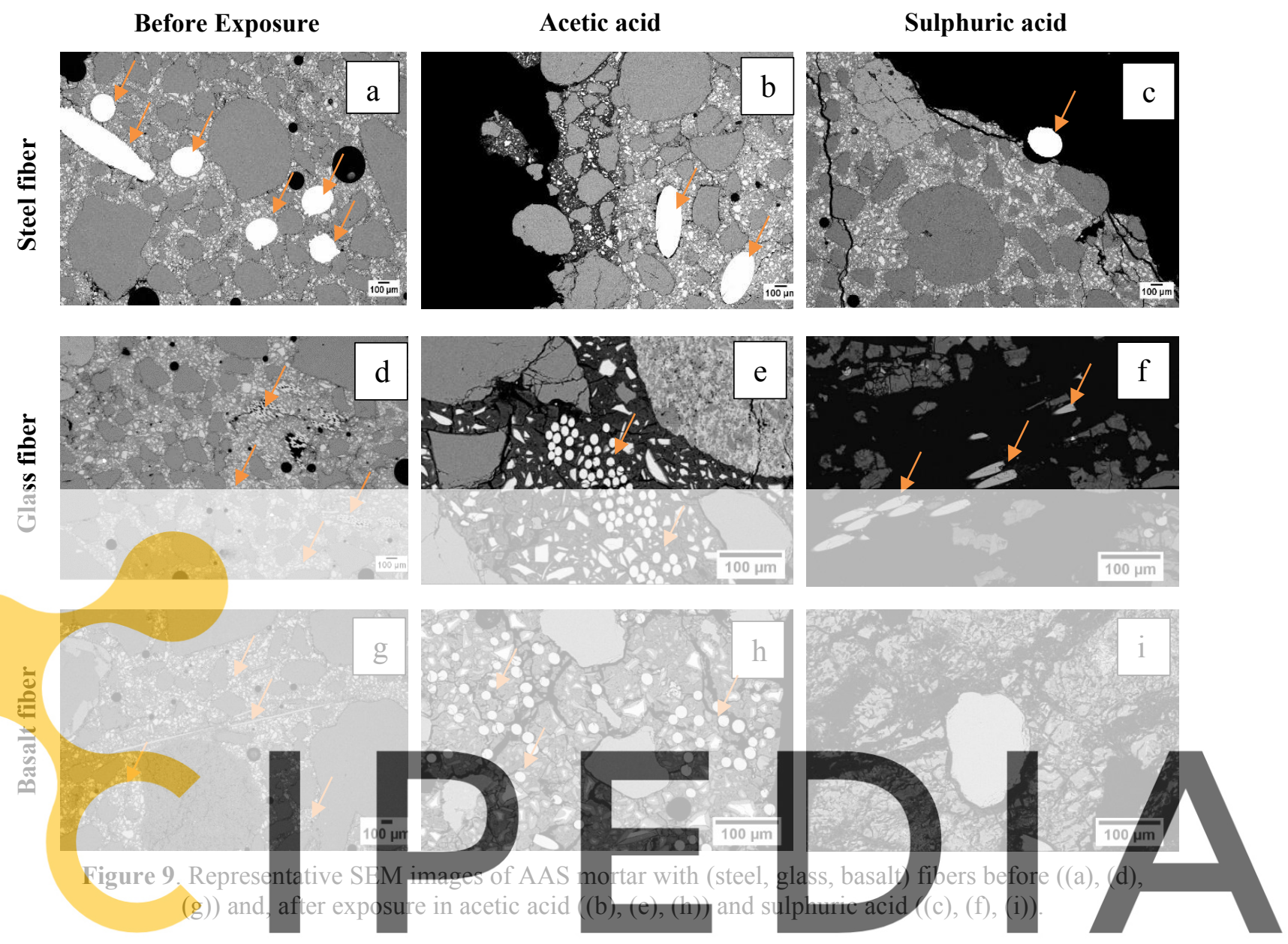

4 Conclusions

Register for free at https//www.scipedia.com to download the version without the watermark

The influence of acetic and sulphuric acid on alkali activated slag mortar is investigated.

Damage of alkali activated slag can be related to the decalcification and dealumination of the calcium silicate gels and the aluminosilicates, respectively. In acetic environment, the formation of calcium acetate acted as a barrier for further diffusion of acid protecting the unaffected core. However, sulphuric acid is corrosive and resulted in disintegration of binder matrix. Presence of fibers improved the performance of AAS mortar in corrosive environment. Residual strength of AAS mortar specimens are $19 \%$ and $7 \%$ higher with the reinforcement of mixed steel fibers $(6$ and $12 \mathrm{~mm}$ ) in acetic and sulphuric acid exposure, respectively. Basalt fibers needs to be coated to use them in highly corrosive environment.

\section{Acknowledgements}

This work was supported by the Finnish Funding Agency for Technology and Innovation (Tekes) (project GEOBIZ, grant number 1105/31/2016). Authors acknowledge Business Finland (grant \# 1215/31/2015) and Academy of Finland (grant \#292526, \#319676 and \#326291) for financial support.

\section{ORCID}

Priyadharshini Perumal: http://orcid.org/0000-0002-7731-2016 
Tirthankar Paul: https://orcid.org/0000-0002-1115-0843

Tero Luukkonen: https://orcid.org/0000-0002-1124-775X

Juha Röning: https://orcid.org/0000-0001-9993-8602

Paivo Kinnunen: https://orcid.org/0000-0001-8490-1890

Mirja Illikainen: https://orcid.org/0000-0001-7545-4574

\section{References}

Aiken, T. A., Kwasny, J., Sha, W. and Soutsos, M. N. (2018). Effect of slag content and activator dosage on the resistance of fly ash geopolymer binders to sulfuric acid attack. Cement and Concrete Research, Elsevier, 111(April), 23-40. doi: 10.1016/j.cemconres.2018.06.011

Bakharev, T. (2005). Resistance of geopolymer materials to acid attack. Cement and Concrete Research, 35(4), 658-670. doi: 10.1016/j.cemconres.2004.06.005

Bernal, S. A., Rodríguez, E. D., Mejía, R. and Gutiérrez, D. (2012). "Performance of alkali-activated slag mortars exposed to acids. Journal of Sustainable Cement-Based Materials, 1(3), 138-151. doi: $10.1080 / 21650373.2012 .747235$

Fernández-Jiménez, A. and Palomo, A. (2009). Chemical durability of geopolymers. Geopolymers: Structures, Processing, Properties and Industrial Applications, Woodhead Publishing Limited. doi: 10.1533/9781845696382.2.167

Heath, A., Paine, K. and McManus, M. (2014). Minimising the global warming potential of clay based geopolymers. Journal of Cleaner Production, 78, 75-83. doi: 10.1016/j.jclepro.2014.04.046

Khan, H. A., Khan, M. S. H., Castel, A. and Sunarho, J. (2018). "Deterioration of alkali-activated mortars exposed to natural aggressive sewer environment." Construction and Building Materials, 186, 577-597. doi: 10.1016/j.conbuildmat.2018.07.137

Kim, B., Boyd, A. J., Kim, H. S. and Lee, S. H. (2015). "Steel and synthetic types of fibre reinforced concrete

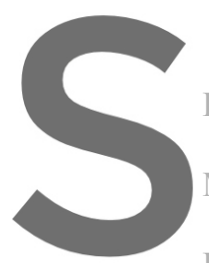
exposed to chemical e 10.1016/j.conbuildnat.2015.0

Koenig, A. and Dehn, F. 147, 226-237. doi:

Mehta, A. and Siddique Construction and Build

Provis, J. L., Muntingh, Y., Lloyd, R.R., Xu, H., Keyte
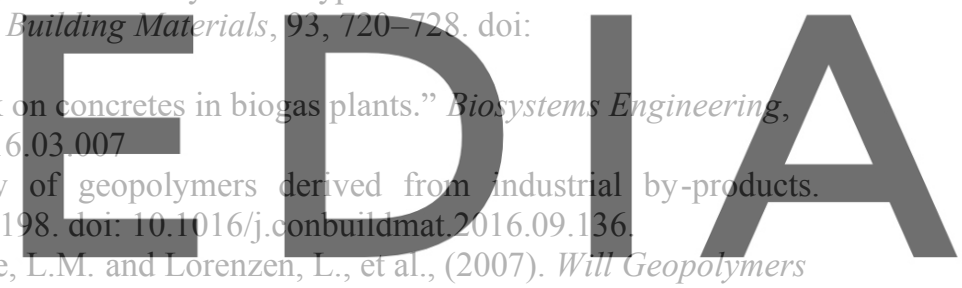
Stand the Test of Time? Developments in Porous, Biological and Geopolymer Ceramics, John Wiley \&

Register for freesalth https//www.scipedia.com to download the version without the watermark

Qi, J., Yu, Y., Wang, L. and Liu, J. (2016). "K*-means: An effective and efficient k-means clustering algorithm." Proceedings - 2016 IEEE International Conferences on Big Data and Cloud Computing, BDCloud 2016, Social Computing and Networking, SocialCom 2016 and Sustainable Computing and Communications, SustainCom 2016, IEEE, 242-249.

Wei, B., Cao, H. and Song, S. (2010). "Tensile behavior contrast of basalt and glass fibers after chemical treatment." Materials and Design, 31(9), 4244-4250. doi: 10.1016/j.matdes.2010.04.009 